\title{
Differential effect of EGFR inhibitors on tamoxifen-resistant breast cancer cells
}

\author{
SANGMIN KIM, JEONGMIN LEE, SOO JIN OH, SEOK JIN NAM and JEONG EON LEE \\ Department of Surgery, Samsung Medical Center, Sungkyunkwan University School of Medicine, \\ Seoul 135-710, Republic of Korea
}

Received January 19, 2015; Accepted April 6, 2015

DOI: $10.3892 /$ or.2015.4116

\begin{abstract}
Although tamoxifen is the most common and effective therapy for treatment of estrogen receptor- $\alpha$ (ER- $\alpha)$ breast cancer patients, resistance of endocrine therapy occurs, either de novo or acquired during therapy. Here, we investigated the clinical value of epidermal growth factor receptor (EGFR) in tamoxifen-resistant (TamR) patients and the differential effect of EGFR inhibitors, neratinib and gefitinib, on TamR breast cancer cell model. The morphology of TamR MCF7 cells showed mesenchymal phenotypes and did not induce cell death by tamoxifen treatment compared with tamoxifen-sensitive (TamS) MCF7 cells. In addition, mesenchymal marker proteins, including $\mathrm{N}$-cadherin (N-cad), fibronectin (FN), and Slug, significantly increased in TamR cells. In contrast, ER- $\alpha$ and E-cadherin (E-cad) were greatly decreased. We also found that the levels of EGFR and HER2 expression were increased in TamR cells. Furthermore, we observed that EGFR expression was directly involved with poor prognosis of tamoxifen-treated breast cancer patients using the GSE1378 date set. Thus, we treated TamR and TamS cells with EGFR inhibitors, neratinib and gefitinib, respectively. Interestingly, neratinib induced apoptotic cell death of TamR but not gefitinib. Cleaved PARP-1 expression was also increased by neratinib treatment in TamR cells. Therefore, we suggest that neratinib may be a potential therapeutic drug for treating TamR breast cancer.
\end{abstract}

\section{Introduction}

Breast cancer is one of the most frequent cancers and the second leading cause of cancer deaths in American women (1). Approximately $70 \%$ of breast cancers express

Correspondence to: Professor Seok Jin Nam or Professor Jeong Eon Lee, Department of Surgery, Samsung Medical Center, Sungkyunkwan University School of Medicine, 50 Irwon-dong, Gangnam-Gu, Seoul 135-710, Republic of Korea

E-mail: sjnam@skku.edu

E-mail:paojlus@hanmail.net

Key words: ER- $\alpha$, EGFR inhibitor, mesenchymal marker protein, tamoxifen resistance estrogen receptors (ER) (1). Estrogen receptor- $\alpha$ (ER- $\alpha$ ) is a very important prognostic marker for selecting an appropriate hormonal therapy $(2,3)$. Breast cancers that express ER and/or the progesterone receptor (PR) are treated with targeted antiestrogen therapy such as tamoxifen $(2,4)$. Adjuvant tamoxifen therapy is effective to prolong disease-free and overall survival of ER-positive breast cancer patients and induces the arrest of tumor progression in $50 \%$ of patients with breast cancer (5). However, although anti-estrogen therapies targeting ER- $\alpha$ prevent disease recurrence in patients with hormone-dependent breast cancer, de novo or acquired resistance occurs during therapy $(6,7)$. Thus, we investigated the effects of new therapeutic drugs for treatment of ER-positive breast cancer patients.

Acquired tamoxifen-resistant (TamR) cells developed from the parental MCF7 breast cancer cells, elevates some epidermal growth factor receptor (EGFR) ligands as well as the level of EGFR and HER2 receptors compared with tamoxifen-responsive MCF7 cells (8). The heterodimerization of enhanced EGFR and HER2 triggers phosphorylation of downstream kinases, including ERK1/2 MAP kinase, Akt, and PKC- $\alpha$ (9-11). In addition, EGF suppresses the expression and activity of the ER- $\alpha$ via the phosphatidylinositol-3-kinase (PI-3K)/Akt pathway in MCF7 cells (12). In contrast, stable transfection of parental MCF-7 cells with a dominant-negative Akt mutant recovers ER- $\alpha$ expression and activity by $70-80 \%$ (12). Generally, an inverse correlation between EGFR and ER- $\alpha$ was maintained at relapse on tamoxifen (13). The acquired expression of EGFR during treatment plays an important role in the development of tamoxifen resistance in human breast cancer (14).

In the present study, we evaluated the effect of EGFR inhibitors, neratinib and gefitinib, in tamoxifen-sensitive (TamS) and TamR cells. TamR cells showed mesenchymal phenotypes and highly expressed mesenchymal marker proteins, including fibronectin (FN), N-cadherin (N-cad), and Slug. In addition, the level of EGFR expression was significantly increased in TamR cells. Interestingly, neratinib, one of EGFR inhibitors, induced cell death of TamR but not gefitinib. Therefore, we demonstrated that neratinib treatment may be a promising therapeutic strategy to overcome tamoxifen resistance.

\section{Materials and methods}

Reagents. Dulbecco's modified Eagle's medium (DMEM) was purchased from Thermo Scientific (Hemel Hempstead, 
UK). Fetal bovine serum (FBS) was purchased from Hyclone (Logan, UT, USA). Phenol red-free DMEM, penicillin $(100 \mathrm{U} / \mathrm{ml})$ and $100 \mathrm{mg} / \mathrm{ml}$ streptomycin were purchased from Life Technologies (Rockville, MD, USA). Neratinib and gefitinib were purchased from Selleck Chemicals (Houston, TX, USA). 4-Hydroxytamoxifen (4-OHT) was purchased from Sigma-Aldrich (St. Louis, MO, USA). Rabbit monoclonal anti-PARP-1, FN, ER- $\alpha$, and Twist were purchased from Epitomics (Burlingame, CA, USA). Epithelial-mesenchymal transition (EMT) antibody sampler [E-cadherin (E-cad), $\mathrm{N}$-cad, and Slug antibodies] was purchased from Cell Signaling Technology (Beverly, MA, USA). The secondary HRP-conjugated antibody and mouse monoclonal anti- $\beta$-actin antibody were purchased from Santa Cruz Biotechnology, Inc. (Santa Cruz, CA, USA). The ECL ${ }^{\text {prime }}$ reagents were purchased from Amersham (Buckinghamshire, UK).

Establishment of TamR MCF7 breast cancer cells. TamS and TamR breast cancer cell lines were kindly provided by Professor Keun Wook Kang (Seoul National University, Seoul, Korea). The TamR was established using methodology reported previously (8). Briefly, to establish TamR, MCF-7 cells were washed with PBS, and the culture medium was changed to phenol red-free DMEM containing 10\% charcoal-stripped steroid-depleted FBS (both from Life Technologies) and $0.1 \mathrm{mM} 4-\mathrm{OHT}$. The cells were continuously exposed to this treatment regimen for 2 weeks, and the 4-OHT concentration was increased gradually up to $3 \mathrm{mM}$ over a 9-month period. Initially, cell growth rates were depressed. However, after exposure to the medium for 9 months, the rate of cell growth increased gradually, indicating the establishment of TamR cells.

Soft agar colony formation assay. TamS and TamR cells were seeded at a density of $5 \times 10^{4}$ cells/well in 6 -well plates in growth medium containing $0.7 \%$ agar $(1.5 \mathrm{ml} /$ well $)$ on top of a layer of growth medium containing $1.4 \%$ agar $(2 \mathrm{ml} /$ well). Growth medium $(500 \mu \mathrm{l})$ with $10 \%$ FBS was added on top of the agar. The cell suspension was plated and cultured in a $37^{\circ} \mathrm{C}$ incubator for 2 weeks. After 2 weeks, viable colonies were stained $0.01 \%$ crystal violet and then were observed using a CK40 inverted microscope (Olympus, Tokyo, Japan).

Flow cytometry analysis (FACS). Apoptosis assays were performed with the Annexin V-fluorescein isothiocyanate (FITC) apoptosis kit-I (BD Pharmingen, San Diego, CA, USA), according to the manufacturers instructions. Briefly, cells $\left(1 \times 10^{6}\right.$ cells $\left./ \mathrm{ml}\right)$ were collected and washed twice with PBS and then resuspended in $500 \mu \mathrm{l}$ of staining solution containing $5 \mu$ l FITC-conjugated Annexin V and propidium iodide (PI). After incubation for $15 \mathrm{~min}$ at room temperature (RT) in the dark, cells were immediately analyzed on a flow cytometer. Apoptotic cells were double-stained with Annexin V and PI and then they were analyzed using the FACS Vantage system (Becton-Dickinson, San Diego, CA, USA). The percentage of cells undergoing apoptosis was determined.

Cell viability. To measure the sensitivities to EGFR inhibitors, neratinib or gefitinib, we analyzed using a Countess Automated Cell Counter (Invitrogen, Carlsbad, CA, USA).
Table I. Primer sequences for analysis of the various gene mRNA expression.

\begin{tabular}{ll}
\hline Gene name & \multicolumn{1}{c}{ Primer sequences } \\
\hline ER- $\alpha$ & F: CGC TAC TGT GCA GTG TGC AAT \\
& R: CCT CAC AGG ACC AGA CTC CAT AA \\
E-cad & F: GGC ACA AAG ATG GGG GCT TC \\
& R: TCA CCA CCT CCA CAG CCA CC \\
N-cad & F: GCA GAT CGG ACC GGA TAC TG \\
& R: TGG GAA TCC GAC GAA TGG \\
FN & F: CCA CCC CCA TAA GGC ATA GG \\
& R: GTA GGG GTC AAA GCA CGA GTC ATC \\
Slug & F: CTG TGG TCC TTG GAG GAG GT \\
& R: GTA GGT GCC AGG GTG GAA AT \\
Twist & F: CAG CTT GCC ATC TTG GAG TC \\
& R: GAC GAC AGC CTG AGC AAC AG \\
EGFR & F: CAT GTC GAT CTT CCA GA \\
& R: GGG ACA GCT TGG ATC ACA CT \\
HER2 & F: CAC TTC AAC CAC AGT GGC AT \\
& R: ATT CAC ATA CTC GGG GA \\
GAPDH & F: ATT GTT GCC ATC AAT GAC CC \\
& R: AGT AGA GGC AGG GAT GAT GT
\end{tabular}

ER- $\alpha$, estrogen receptor- $\alpha$; E-cad, E-cadherin; N-cad, N-cadherin; FN, fibronectin; EGFR, epidermal growth factor receptor; F, forward; R, reverse.

Briefly, TamS and TamR cells $\left(5 \times 10^{4}\right.$ cells/well) were seeded onto 6-well plates. TamS and TamR cells were incubated in phenol red-free DMEM containing 10\% charcoal-stripped steroid-depleted FBS without $3 \mu \mathrm{M} 4-\mathrm{OHT}$ and $2.5 \mu \mathrm{M}$ neratinib or gefitinib, respectively for $24 \mathrm{~h}$.

Western blotting. The cell lysates were used in the immunoblot analysis for PARP-1, E-cad, N-cad, FN, Twist, Slug, EGFR, HER 2, and $\beta$-actin. The proteins were boiled for $5 \mathrm{~min}$ in Laemmli sample buffer and then electrophoresed on $8 \%$ or $10 \%$ sodium dodecyl sulfate-polyacrylamide gel electrophoresis gels. The proteins were transferred to PVDF membranes, and the membranes were blocked with $10 \%$ skim milk in TBS with $0.01 \%$ Tween-20 for $15 \mathrm{~min}$. The blots were incubated with anti-PARP-1, ER- $\alpha$, E-cad, N-cad, FN, Twist, Slug, EGFR, HER2, and $\beta$-actin antibodies (1:1,000 dilution) in 1\% TBS/T buffer $\left(0.01 \%\right.$ Tween-20 in TBS) at $4^{\circ} \mathrm{C}$ overnight. The blots were washed 3 times in TBS with $0.01 \%$ Tween-20, and they were subsequently incubated with anti-rabbit HRP-conjugated antibody (1:5,000 dilution) in TBS/T buffer. After 1-h incubation at RT, the blots were washed 3 times and ECL ${ }^{\text {prime }}$ reagents were applied for further development.

Microarray data analysis. We downloaded expression data from a public database (GSE1378) and analyzed the clinical value of EGFR in tamoxifen-treated breast cancer patients.

Real-time polymerase chain reaction (RT-PCR). Total RNA was extracted from the cells using TRIzol reagent (Invitrogen), according to the manufacturers instructions. Isolated RNA 
A

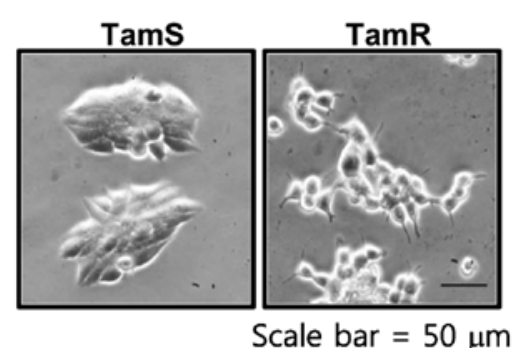

B

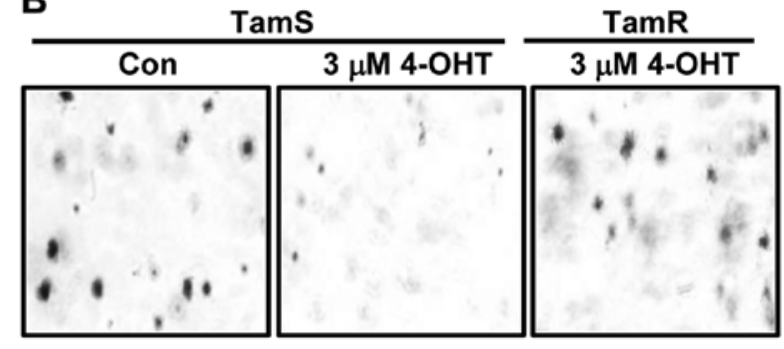

C

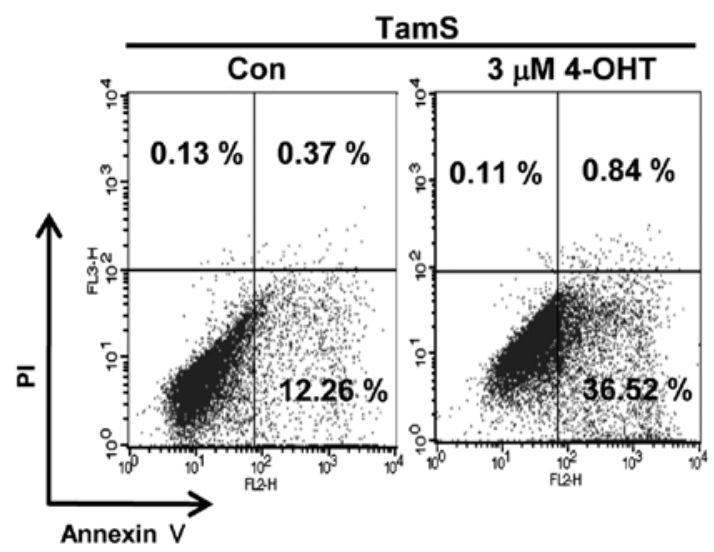

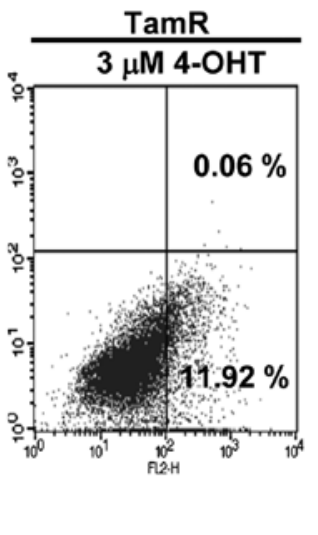

Figure 1. Differential characteristics of TamS and TamR MCF7 breast cancer cells. (A) Cell morphology of established TamS and TamR MCF7 cells. (B) Established TamS and TamR MCF7 cells were seeded in a 6-well soft agar plate with or without $3 \mu \mathrm{M} 4-\mathrm{OHT}$ for 2 weeks. (C) Established TamS and TamR MCF7 cells were seeded with or without $3 \mu \mathrm{M} 4$-OHT for $24 \mathrm{~h}$. Apoptotic cells were analyzed by flow cytometry. Results are representative of 3 independent experiments. Con, control; TamR, tamoxifen-resistant; TamS, tamoxifen-sensitive; 4-OHT, 4-Hydroxytamoxifen.

samples were then used for RT-PCR. Samples $(1 \mu \mathrm{g}$ total RNA) were reverse-transcribed into cDNA in $20-\mu 1$ reaction volumes using a First Strand cDNA synthesis kit for RT-PCR, according to the manufacturer's instructions (MBI Fermentas, Hanover, MD, USA).

Gene expression was quantified by real-time PCR using a SensiMix SYBR kit (Bioline Ltd., London, UK) and $100 \mathrm{ng}$ of cDNA per reaction. The sequences of the primer sets used for this analysis are shown in Table I. An annealing temperature of $60^{\circ} \mathrm{C}$ was used for all primers. PCRs were performed in a standard 384-well plate format with an ABI 7900HT Real-Time PCR detection system (Applied Biosystems, Foster City, CA, USA). The raw threshold cycle $\left(\mathrm{C}_{\mathrm{T}}\right)$ value was first normalized to the housekeeping gene for each sample to obtain $\Delta \mathrm{C}_{\mathrm{T}}$. The normalized $\Delta \mathrm{C}_{\mathrm{T}}$ was then calibrated to the control cell samples to obtain $\Delta \Delta \mathrm{C}_{\mathrm{T}}$. All cDNA samples were analyzed in 3 independent experiments.

Statistical analysis. Statistical significance was determined using the Student's t-test. Results are presented as means \pm standard errors. All P-values are two-tailed, and differences were considered significant at $\mathrm{P}<0.05$.

\section{Results and Discussion}

To establish a new therapeutic strategy for treatment of TamR breast cancer, we analyzed differential characteristics of TamS and TamR breast cancer cell lines. In a previous study, breast cancer cell lines could be classified into four distinct morphological groups referred to as round, mass, grape-like, and stellate (15). As shown in Fig. 1A, we also observed the morphological difference between TamS and TamR cells. TamS cells stacked up to form colonies and TamR cells scattered, in loosely-packed colonies, and had many branches. In addition, to assess the tumorigenicity degree of TamS and TamR cells, we examined the effect of tamoxifen on the ability of cells to form colonies using soft agar colony formation assays. The colonies of TamS cells were greatly decreased by $3 \mu \mathrm{M}$ tamoxifen treatment while TamR cells still maintained colonies (Fig. 1B). We also measured the apoptotic cell death of TamS and TamR cells by tamoxifen. Cells were treated with $3 \mu \mathrm{M}$ tamoxifen for $24 \mathrm{~h}$ and examined by Annexin V/PI staining. As shown in Fig. 1C, the apoptotic cell population of TamS cells was significantly increased by 3 -fold of control level. However, the apoptotic cell population of TamR cells was similar to control of TamS (Fig. 1C).

In a previous study, tamoxifen resistance was associated with enhanced cell motility (16). Antiestrogens also promote cellular invasion and motility in TamR breast cancer cells (17). In addition, TamR MCF7 cells promote EMT-like behavior and inhibition of EGFR in these cells augments cell-cell adhesion (18). Thus, we also investigated the relationship between the morphological change of TamR cells and EMT. Although the level of Twist expression did not change, the expression levels of mesenchymal marker proteins such as N-cad, FN, and Slug, was significantly increased in TamR cells (Fig. 2A). 
A

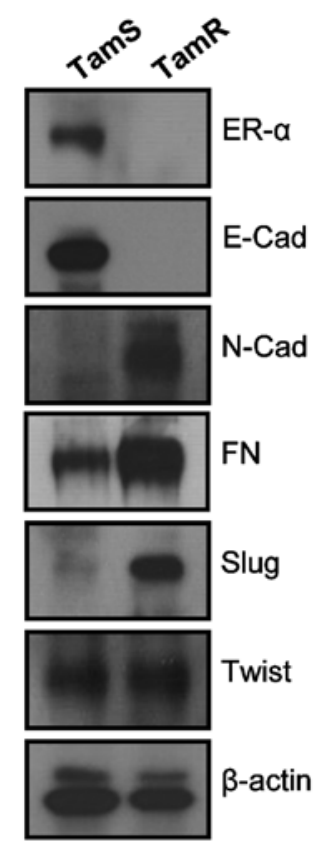

B
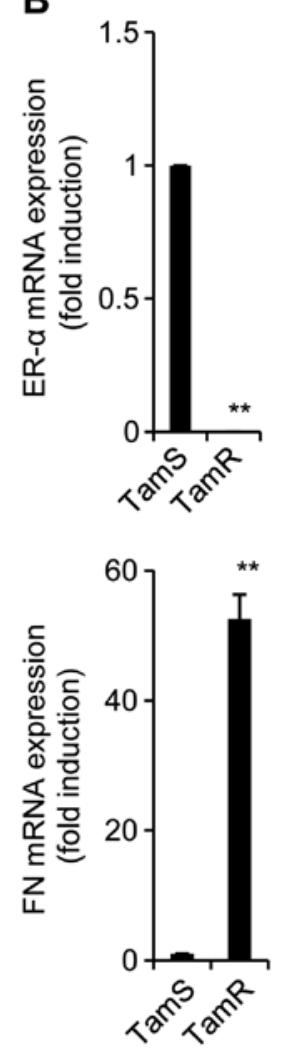
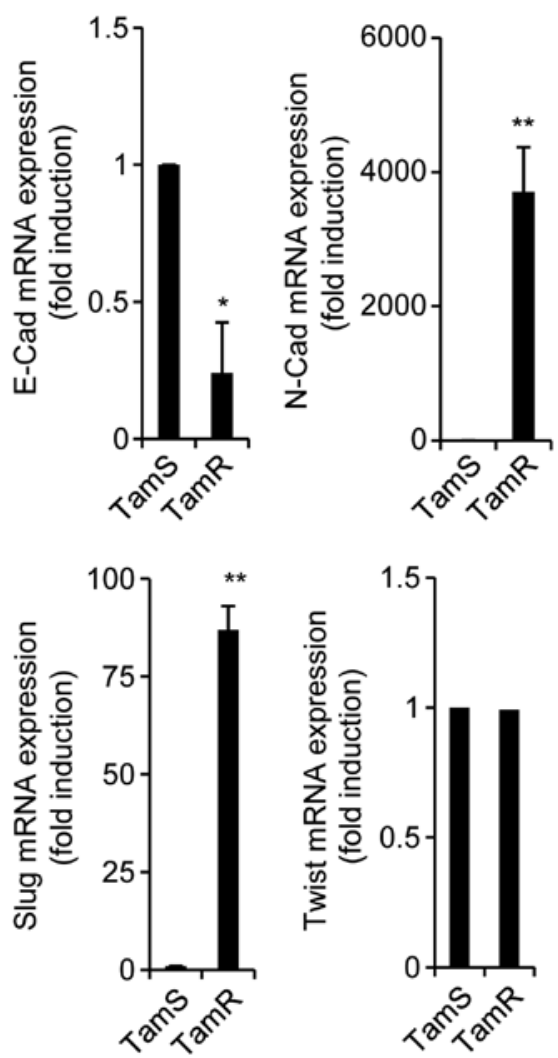

Figure 2. TamR cells highly express mesenchymal marker proteins. Established TamS and TamR cells were seeded in a 6 -well plate with or without $3 \mu \mathrm{M}$ 4-OHT for $24 \mathrm{~h}$. After $24 \mathrm{~h}$, TamS and TamR cells were harvested for detection of protein expressions (A) and mRNA expressions (B). ER- $\alpha$, E-cad, N-cad, FN, Slug, Twist, and $\beta$-actin expression was analyzed by western blotting (A) and real-time PCR (B), respectively. Results are representative of 3 independent experiments. Values shown are means \pm standard errors. ${ }^{*} \mathrm{P}<0.05,{ }^{* *} \mathrm{P}<0.01$ vs. TamS. TamR, tamoxifen-resistant; TamS, tamoxifen-sensitive; $4-\mathrm{OHT}$, 4-Hydroxytamoxifen; ER- $\alpha$, estrogen receptor- $\alpha$; E-cad, E-cadherin; N-cad, N-cadherin; FN, fibronectin.

In contrast, ER- $\alpha$ and E-cad expression (epithelial marker protein) was decreased in TamR cells (Fig. 2A). Our results also showed that the mRNA expression of these proteins was similar with the patterns of proteins expression (Fig. 2B). Therefore, we demonstrated that tamoxifen resistance is associated with the severe reduction of ER- $\alpha$ expression and the induction of EMT.

We observed that the levels of EGFR and HER2 proteins and mRNA expression were dramatically enhanced in TamR cells (Fig. 3A and B). The levels of EGFR and HER2 mRNA expression were increased by 14.0-fold and 3.4-fold of control level, respectively (Fig. 3A and B). Consistent with our data, the induction of growth factor receptors, such as EGFR and HER2 has been implicated in acquired resistance to endocrine therapy $(19,20)$. Snail overexpressed cells are resistant to tamoxifen and increase the level of EGFR expression (21). Next, we analysed the clinical value of EGFR expression in tamoxifen-treated breast cancer patients using public microarray datasets (GSE1378). Interestingly, patients with high EGFR expression levels showed significantly shorter relapse-free survival time (Fig. $3 \mathrm{C}, \mathrm{P}=0.000034$ ). These results suggest that the level of EGFR expression plays an important role in tamoxifen resistance.

We investigated the effect of EGFR inhibitors, neratinib and gefitinib, in TamS and TamR cells. Neratinib (HKI-272, Pfizer; Puma Biotechnology) is a pan-HER receptor tyrosine kinase inhibitor (EGFR, HER2 and HER4) and binds irre- versible to these kinases (22). Gefitinib (Iressa; AstraZeneca Pharmaceuticals) is the first selective inhibitor of EGFR tyrosine kinase domain and is responsible for activating anti-apoptotic pathways in non-small cell lung cancers (23). As shown in Fig. 4A, gefitinib did not affect the viabilities of TamS and TamR cells. The $\mathrm{IC}_{50}$ value of gefitinib was $\sim 20 \mu \mathrm{M}$ concentration. However, neratinib significantly decreased the viability of TamR cells at $0.43 \mu \mathrm{M}$ concentration but the viability of TamS cells did not change by $7.3 \mu \mathrm{M}$ neratinib treatment (Fig. 4B). Therefore, we demonstrated that neratinib prevents more effectively EGFR and HER2 signaling pathways as well as triggers apoptotic cell death of TamR cells.

To evaluate the tumorigenicity of TamR cells by EGFR inhibitors, we examined the effect of neratinib and gefitinib on the ability of cells to form colonies using soft agar colony formation assays. As shown in Fig. 5A, the colonies of TamR cells were completely decreased by $2.5 \mu \mathrm{M}$ neratinib treatment while gefitinib-treated TamR cells still maintained colonies. Furthermore, one of apoptosis marker proteins, cleaved PARP-1 expression was also dramatically increased by neratinib treatment (Fig. 5B). Finally, we also measured the apoptotic cell death of TamR cells by neratinib and gefitinib. Cells were treated with $2.5 \mu \mathrm{M}$ neratinib and gefitinib, respectively for $24 \mathrm{~h}$. The apoptotic cell population of TamR cells by neratinib was significantly increased by 4 -fold of control level (Fig. 5C). However, the apoptotic cell population by gefitinib was slightly increased (Fig. 5C). Therefore, we suggest 
A

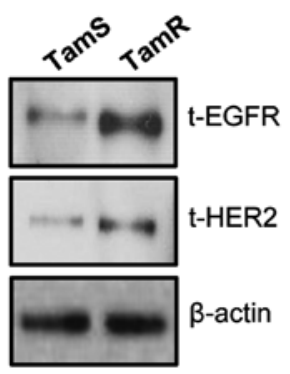

B

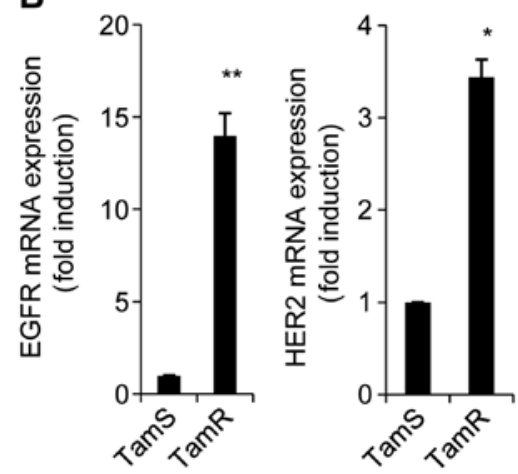

C

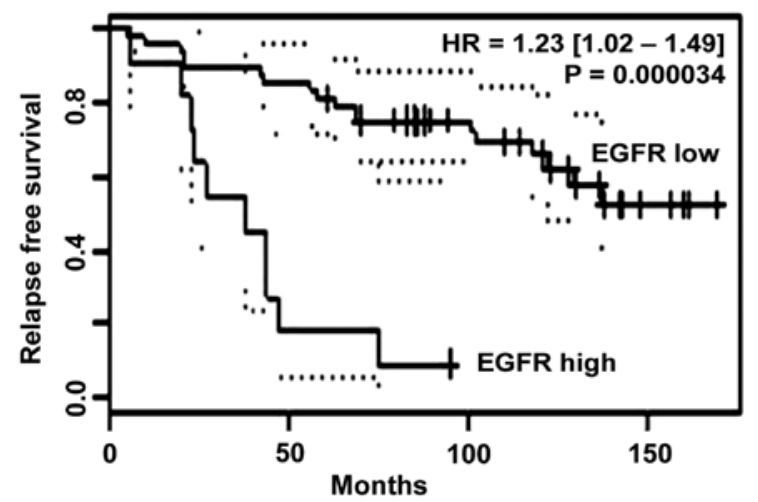

Figure 3. The levels of EGFR and HER2 expression are significantly increases in TamR breast cancer. After $24 \mathrm{~h}$, TamS and TamR cells were harvested for detection of protein (A) and mRNA expression (B). EGFR, HER2 and $\beta$-actin expression was analyzed by western blotting (A) and real-time PCR (B), respectively. (C) EGFR gene expression correlated with poor relapse-free survival of tamoxifen-treated patients. The P-value shown was computed by log-rank test. Results are representative of 3 independent experiments. Values shown are means \pm standard errors. ${ }^{*} \mathrm{P}<0.05,{ }^{* *} \mathrm{P}<0.01$ vs. TamS. EGFR, epidermal growth factor receptor; TamR, tamoxifen-resistant; TamS, tamoxifen-sensitive.

A
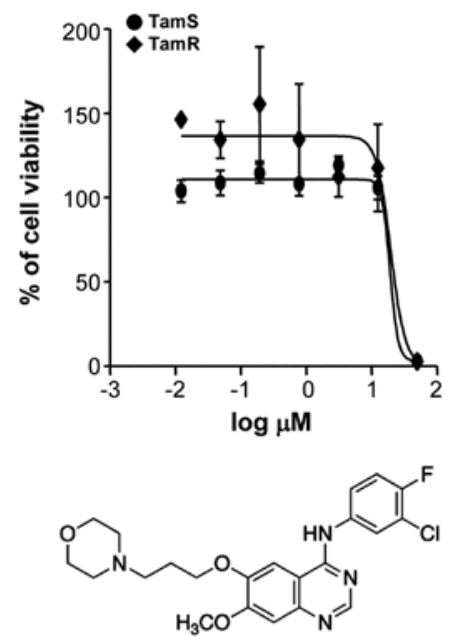

B
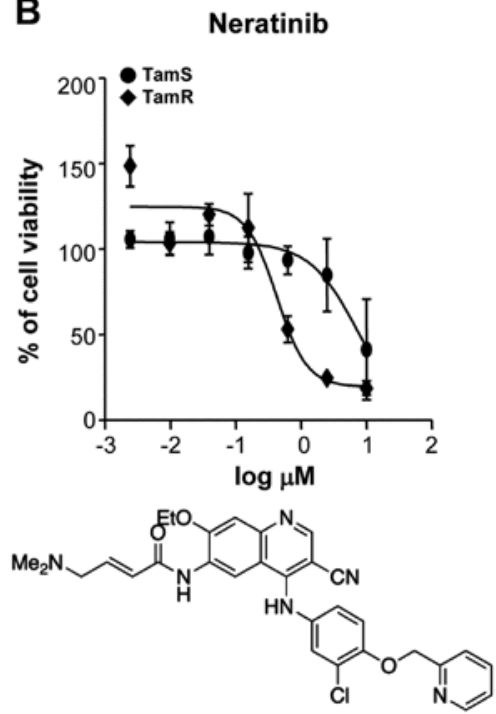

\begin{tabular}{|c|c|c|c|}
\hline \multirow{2}{*}{ Drug } & \multirow{2}{*}{ Target } & \multicolumn{2}{|c|}{$\mathrm{IC}_{50}(\mu \mathrm{M})$} \\
\cline { 3 - 4 } Gefitinib & EGFR & 19.2 & 19.7 \\
\hline Neratinib & $\begin{array}{c}\text { EGFR, HER2, } \\
\text { HER4 }\end{array}$ & 7.3 & 0.43 \\
\hline
\end{tabular}

Figure 4. TamR cells are induced cell death by neratinib treatment but not by gefitinib. (A and B) TamS and TamR cells were seeded in $3 \mu \mathrm{M} 4-\mathrm{OHT}$ free culture media and then treated with the indicated concentration of gefitinib (A) or neratinib (B) for $24 \mathrm{~h}$. After $24 \mathrm{~h}$, viabilities of TamS and TamR cells were analyzed using a Countess Automated Cell Counter. Results are representative of 3 independent experiments. TamR, tamoxifen-resistant; TamS, tamoxifensensitive; 4-OHT, 4-Hydroxytamoxifen. 
A

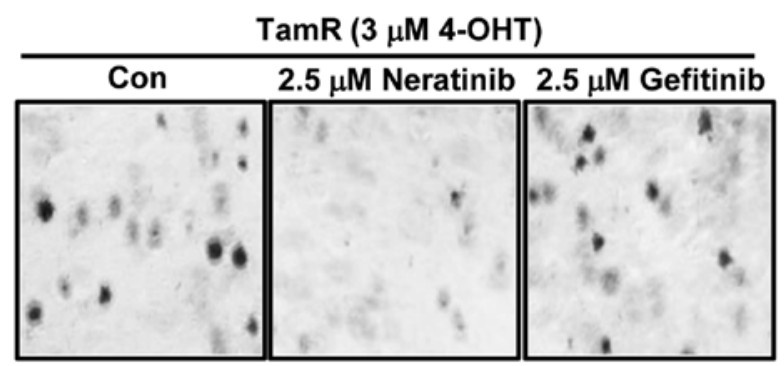

B TamR

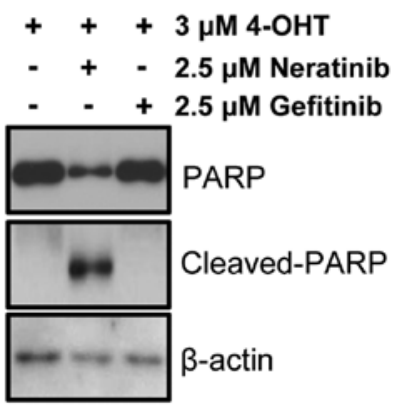

C

$\operatorname{TamR}(3 \mu \mathrm{M}$ 4-OHT)

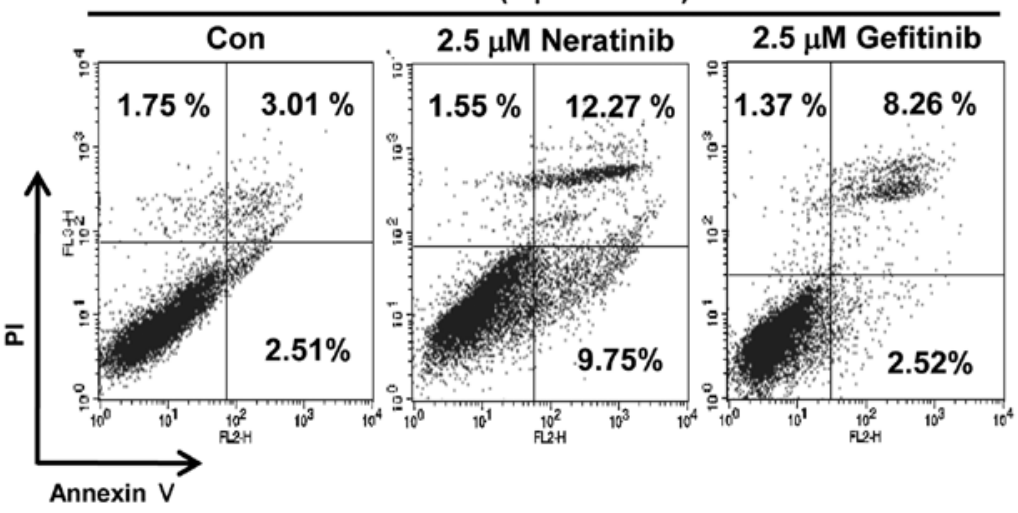

Figure 5. TamR cells induce apoptotic cell death by neratinib. (A) TamR cells were seeded in a 6-well soft agar plate with or without $2.5 \mu \mathrm{M}$ neratinib or gefitinib for 2 weeks. (B) TamR cells were seeded in a 6-well plate with or without $2.5 \mu \mathrm{M}$ neratinib or gefitinib for $24 \mathrm{~h}$. After 24 h, TamR cells were harvested for detection of protein expressions. PARP-1 (mature and cleaved forms) and $\beta$-actin expression was analyzed by western blotting. (C) TamR cells were seeded with or without $2.5 \mu \mathrm{M}$ neratinib or gefitinib for $24 \mathrm{~h}$. Apoptotic cells were analyzed by flow cytometry. Results are representative of 3 independent experiments. Con, control; TamR, tamoxifen-resistant.

that neratinib may be a new therapeutic drug for treatment of TamR breast cancer.

\section{Acknowledgements}

This research was supported by a grant of the Korea Health Technology R\&D Project through the Korea Health Industry Development Institute (KHIDI), funded by the Ministry of Health and Welfare, Republic of Korea (HI14C3418) and by the Samsung Biomedical Research Institute grant (SMX1131701).

\section{References}

1. Jemal A, Siegel R, Xu J and Ward E: Cancer statistics, 2010. CA Cancer J Clin 60: 277-300, 2010.

2. Osborne CK, Zhao H and Fuqua SA: Selective estrogen receptor modulators: structure, function, and clinical use. J Clin Oncol 18 3172-3186, 2000.

3. Harvey JM, Clark GM, Osborne CK and Allred DC: Estrogen receptor status by immunohistochemistry is superior to the ligand-binding assay for predicting response to adjuvant endocrine therapy in breast cancer. J Clin Oncol 17: 1474-1481, 1999.

4. Herynk MH and Fuqua SA: Estrogen receptors in resistance to hormone therapy. Adv Exp Med Biol 608: 130-143, 2007.

5. Ali S and Coombes RC: Endocrine-responsive breast cancer and strategies for combating resistance. Nat Rev Cancer 2: 101-112, 2002.

6. Kurebayashi J: Endocrine-resistant breast cancer: underlying mechanisms and strategies for overcoming resistance. Breast Cancer 10: 112-119, 2003.
7. Schiff R, Massarweh S, Shou J and Osborne CK: Breast cancer endocrine resistance: how growth factor signaling and estrogen receptor coregulators modulate response. Clin Cancer Res 9: 447S-454S, 2003.

8. Knowlden JM, Hutcheson IR, Jones HE, Madden T, Gee JM, Harper ME, Barrow D, Wakeling AE and Nicholson RI: Elevated levels of epidermal growth factor receptor/c-erbB2 heterodimers mediate an autocrine growth regulatory pathway in tamoxifen-resistant MCF-7 cells. Endocrinology 144: 1032-1044, 2003.

9. Kim S, Lee J, Lee SK, Bae SY, Kim J, Kim M, Kil WH, Kim SW, Lee JE and Nam SJ: Protein kinase C- $\alpha$ downregulates estrogen receptor- $\alpha$ by suppressing c-Jun phosphorylation in estrogen receptor-positive breast cancer cells. Oncol Rep 31: 1423-1428, 2014.

10. Benz CC, Scott GK, Sarup JC, Johnson RM, Tripathy D, Coronado E, Shepard HM and Osborne CK: Estrogen-dependent, tamoxifen-resistant tumorigenic growth of MCF-7 cells transfected with HER2/neu. Breast Cancer Res Treat 24: 85-95, 1992.

11. Li Z, Wang N, Fang J, Huang J, Tian F, Li C and Xie F: Role of PKC-ERK signaling in tamoxifen-induced apoptosis and tamoxifen resistance in human breast cancer cells. Oncol Rep 27: 1879-1886, 2012

12. Martin MB, Franke TF, Stoica GE, Chambon P, Katzenellenbogen BS, Stoica BA, McLemore MS, Olivo SE and Stoica A: A role for Akt in mediating the estrogenic functions of epidermal growth factor and insulin-like growth factor I. Endocrinology 141: 4503-4511, 2000.

13. deFazio A, Chiew YE, McEvoy M, Watts CK and Sutherland RL: Antisense estrogen receptor RNA expression increases epidermal growth factor receptor gene expression in breast cancer cells. Cell Growth Differ 8: 903-911, 1997.

14. Newby JC, Johnston SR, Smith IE and Dowsett M: Expression of epidermal growth factor receptor and c-erbB2 during the development of tamoxifen resistance in human breast cancer. Clin Cancer Res 3: 1643-1651, 1997. 
15. Kenny PA, Lee GY, Myers CA, Neve RM, Semeiks JR, Spellman PT, Lorenz K, Lee EH, Barcellos-Hoff MH, Petersen OW, et al: The morphologies of breast cancer cell lines in three-dimensional assays correlate with their profiles of gene expression. Mol Oncol 1: 84-96, 2007.

16. Zhou C, Zhong Q, Rhodes LV, Townley I, Bratton MR, Zhang Q, Martin EC, Elliott S, Collins-Burow BM, Burow ME, et al Proteomic analysis of acquired tamoxifen resistance in MCF-7 cells reveals expression signatures associated with enhanced migration. Breast Cancer Res 14: R45, 2012.

17. Borley AC, Hiscox S, Gee J, Smith C, Shaw V, Barrett-Lee P and Nicholson RI: Anti-oestrogens but not oestrogen deprivation promote cellular invasion in intercellular adhesion-deficient breast cancer cells. Breast Cancer Res 10: R103, 2008.

18. Hiscox S, Jiang WG, Obermeier K, Taylor K, Morgan L, Burmi R, Barrow D and Nicholson RI: Tamoxifen resistance in MCF7 cells promotes EMT-like behaviour and involves modulation of betacatenin phosphorylation. Int J Cancer 118: 290-301, 2006.

19. Hutcheson IR, Knowlden JM, Madden TA, Barrow D, Gee JM, Wakeling AE and Nicholson RI: Oestrogen receptor-mediated modulation of the EGFR/MAPK pathway in tamoxifen-resistant MCF-7 cells. Breast Cancer Res Treat 81: 81-93, 2003.
20. Kurokawa H, Lenferink AE, Simpson JF, Pisacane PI, Sliwkowski MX, Forbes JT and Arteaga CL: Inhibition of HER2/neu (erbB-2) and mitogen-activated protein kinases enhances tamoxifen action against HER2-overexpressing, tamoxifen-resistant breast cancer cells. Cancer Res 60: 5887-5894, 2000.

21. Jiang Y, Zhao X, Xiao Q, Liu Q, Ding K, Yu F, Zhang R, Zhu T and Ge G: Snail and Slug mediate tamoxifen resistance in breast cancer cells through activation of EGFR-ERK independent of epithelial-mesenchymal transition. J Mol Cell Biol 6: 352-354, 2014.

22. Rabindran SK, Discafani CM, Rosfjord EC, Baxter M, Floyd MB, Golas J, Hallett WA, Johnson BD, Nilakantan R, Overbeek E, et al: Antitumor activity of HKI-272, an orally active, irreversible inhibitor of the HER-2 tyrosine kinase. Cancer Res 64: 3958-3965, 2004

23. Sordella R, Bell DW, Haber DA and Settleman J: Gefitinib-sensitizing EGFR mutations in lung cancer activate anti-apoptotic pathways. Science 305: 1163-1167, 2004. 